\title{
Patterning nonisometric origami in nematic elastomer sheets
}

\author{
Paul Plucinsky ${ }^{\mathrm{a}}$, Benjamin A. Kowalski ${ }^{\mathrm{b}}$, Timothy J. White ${ }^{\mathrm{b}}$, and Kaushik Bhattacharya ${ }^{\mathrm{c}}$ \\ ${ }^{a}$ Aerospace and Engineering Mechanics, University of Minnesota \\ ${ }^{b}$ Materials and Manufacturing Directorate, Air Force Research Lab \\ ${ }^{c}$ Engineering and Applied Sciences, California Institute of Technology
}

December 14, 2017

\begin{abstract}
Nematic elastomers dramatically change their shape in response to diverse stimuli including light and heat. In this paper, we provide a systematic framework for the design of complex three dimensional shapes through the actuation of heterogeneously patterned nematic elastomer sheets. These sheets are composed of nonisometric origami building blocks which, when appropriately linked together, can actuate into a diverse array of three dimensional faceted shapes. We demonstrate both theoretically and experimentally that: 1) the nonisometric origami building blocks actuate in the predicted manner, 2) the integration of multiple building blocks leads to complex multi-stable, yet predictable, shapes, 3) we can bias the actuation experimentally to obtain a desired complex shape amongst the multi-stable shapes. We then show that this experimentally realized functionality enables a rich possible design landscape for actuation using nematic elastomers. We highlight this landscape through theoretical examples, which utilize large arrays of these building blocks to realize a desired three dimensional origami shape. In combination, these results amount to an engineering design principle, which we hope will provide a template for the application of nematic elastomers to emerging technologies.
\end{abstract}

\section{Introduction}

The seamless integration of function and form promises to spur innovation in technologies ranging from MEMS and NEMS devices (e.g., with novel electrical, electromagnetic and energy functionality), reconfigurable and soft robotics, wearable electronics, and compliant bio-medical devices [6, 9, 10, 14, 24, 33]. This integration can be facilitated by incorporating soft active materials into thin or slender structures to program complex three dimensional shapes not easily achieved by conventional means of manufacturing. This is not without its challenges: The coupling of nonlinearities - at the material level and at the structural level-makes a salient and general theory of design with these systems a trying task. Even more, bridging the gap between an idealized theory and what is capable (and practical) experimentally offers a different, but equally important, set of challenges. In this work, we address many of these challenges in the context of active patterned nematic elastomer sheets by developing a systematic framework for actuating complex shapes in these systems that is grounded in both first principles theory and experimental capability.

Nematic elastomers combine the elasticity of a soft, highly deformable polymer network with the orientational ordering of liquid crystalline monomer units. This results in a solid with dramatic shape-changing response to temperature change and other stimuli [31, 32]: At low temperatures, the liquid crystals prefer being aligned (in some average sense), with the orientation of this alignment 


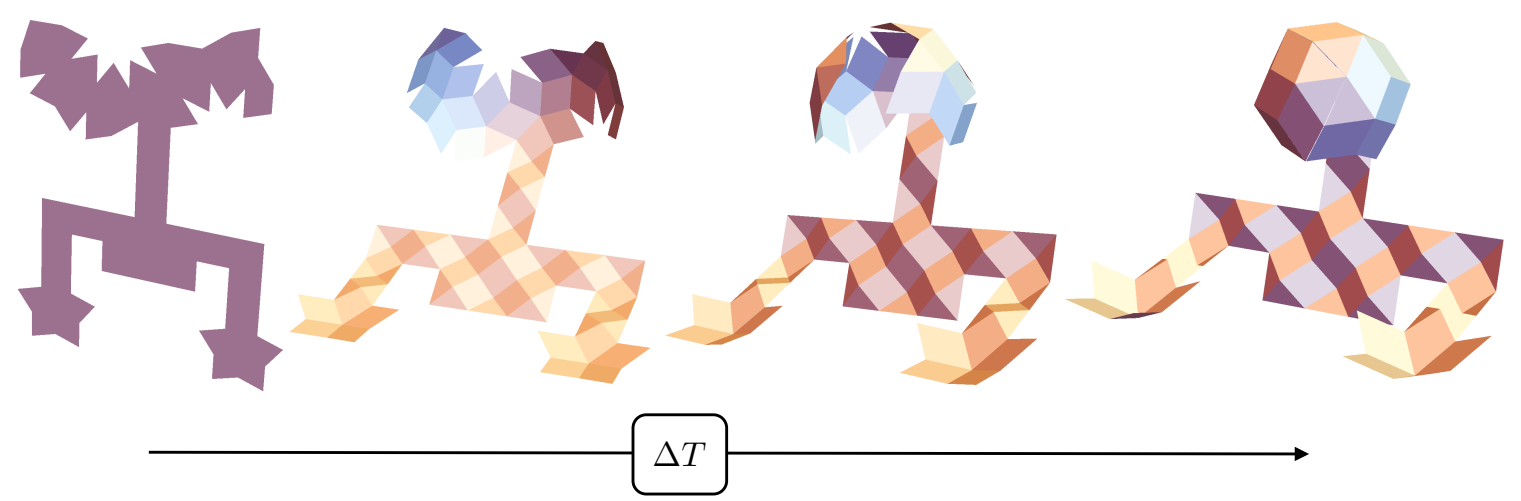

Figure 1: A "humanoid" soft robot.

described locally by a unit vector $\mathbf{n}_{0}$ called the director. Upon heating, however, the molecular order is disrupted, and this gives rise to a strongly anisotropic macroscopic deformation since the polymer network is intrinsically coupled to this order. Typically, the elastomer contracts along the director and expands transversely.

Building on key ideas in the study of non-Euclidean plates [4, 11, 26], Modes et al. [15, 17] recognized that by programming the director heterogeneously in the plane of a thin sheet, stimulation results in inhomogeneous shape-change that in turn drives complex three dimensional shapes. Indeed, they showed theoretically how patterning azimuthal and radial director profiles about a defect enabled conical and saddle-like shapes upon actuation. These were later realized experimentally - first by de Haan et al. [3] in nematic glass sheets and later by Ware et al. [27] in nematic elastomers. Even more, the latter work made possible the prescription of an arbitrary planar director profile in a thin sheet (i.e., through voxelated LCEs), bringing questions of designable actuation to the forefront. Further, since the entire sheet participates in the actuation, it is extremely robust and capable large actuation force and energy. This has motivated the study of other more complex patterns [1, 18, 20, 21, 22, 23]. Of particular interest to this work is the class of nonisometric origami patterns, in which the director is piecewise uniform in the plane. These enable the design of complex faceted (origami) shapes from simple building blocks [22, 23.

However, all the complex patterns described in these works [1, 3, 15, 17, 18, 20, 21, 22, 23, 27. suffer from having multiple, energetically degenerate actuated configurations. For example, the cone can actuate either up or down. This is compounded by the fact that, as patterns are made more complex, the number of multi-stable configurations increases. We illustrate this in Figure 2 , showing a nonisometric origami pattern comprising a joined pair of actuating pyramids. (The pyramid design is discussed in more detail below). This sample exhibits two non-trivially distinct configurations which are mechanically bi-stable. Thus, in order to deterministically actuate the sheet into a single desired configuration, this degeneracy must be broken, ideally in a way that allows us to arbitrarily program individual folds to actuate up or down as prescribed.

One obvious strategy for breaking degeneracies is to exploit the twisted nematic profile, where the planar director varies through the thickness of the sheet. These profiles are known to generate a spontaneous curvature upon actuation which results in complex bending deformations both in isolated strips [19, 25, 29] and when integrated into larger patterned sheets [7, 8, 16]. However, twisted nematics induce a spontaneous Gauss curvature, which can lead to undesirable anti-clastic bending. Even further, Gauss' Theorema Egregium states that a change in Gauss curvature is necessarily accompanied by a stretch. Consequently, this mode of actuation always results in mechanical frustrations in the sheet that influence the robustness of actuation [30]. So, this strategy has proven 


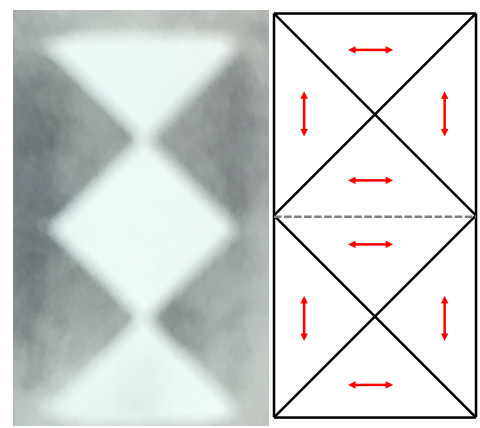

(a)

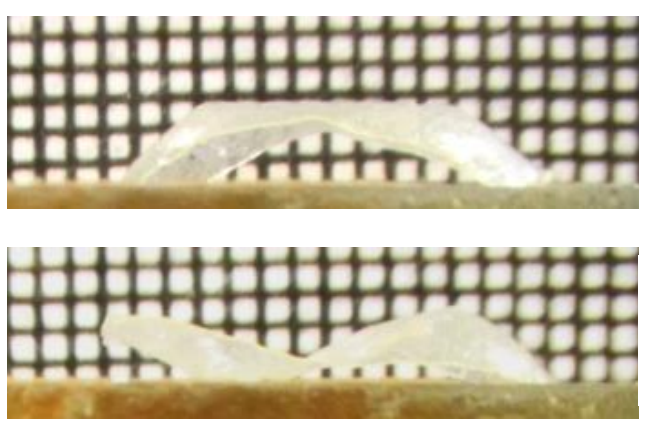

(b)

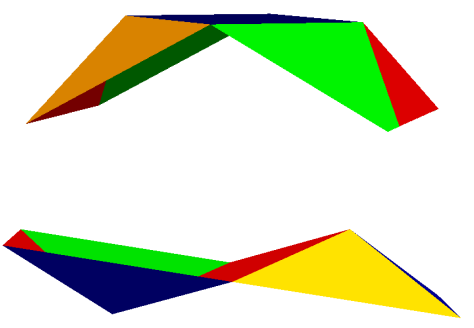

(c)

Figure 2: (a) The design and experimental realization for two linked symmetric four-faced junctions (right and left respectively). (b) The sheet actuates into either of two degenerate and mechanically bi-stable configurations. (c) These configurations are predicted by theory, as discussed below.

effective only when applied in narrow regions. Unfortunately, this restricts the actuation force and energy.

Our key idea, then, is a hybrid approach in which we modify nonisometric origami so that large regions of uniform director are joined by narrow boundary regions of slight nematic twist. In doing this, we achieve a robust actuation, with large actuation force and energy, but also with a desired bias. To develop these ideas, we provide explicit and quantitative design guidance for introducing the bias but also suppressing unwanted anti-clastic bending at the boundary regions. We then incorporate this bias into simple building blocks of nonisometric origami, and show that this is an experimentally accessible design strategy. Finally, we show how these building blocks can be composed systematically to achieve a design landscape with vast potential. One such example is highlighted in Figure 1 .

\section{Notation}

We will be dealing with vector quantities, both in two and three dimensions. Sometimes these will be in the same equation. Thus, we use tilde as a means of distinguishing these quantities. For instance, we use $\mathbf{v}$ for a vector on $\mathbb{R}^{3}$ and $\tilde{\mathbf{v}}$ for a vector on $\mathbb{R}^{2}$; we define the standard basis on $\mathbb{R}^{3}$ as $\left\{\mathbf{e}_{1}, \mathbf{e}_{2}, \mathbf{e}_{3}\right\} \in \mathbb{S}^{2}$ and the standard basis on $\mathbb{R}^{2}$ as $\left\{\tilde{\mathbf{e}}_{1}, \tilde{\mathbf{e}}_{2}\right\} \in \mathbb{S}^{1}$; we set the Cartesian coordinates $\mathbf{x}:=x_{1} \mathbf{e}_{1}+x_{2} \mathbf{e}_{2}+x_{3} \mathbf{e}_{3}$ and $\tilde{\mathbf{x}}:=x_{1} \tilde{\mathbf{e}}_{1}+x_{2} \tilde{\mathbf{e}}_{2}$; we define the three dimensional gradient as $\nabla$ (with respect to $\mathbf{x}$ ) and the planar gradient as $\tilde{\nabla}$ (with respect to $\tilde{\mathbf{x}}$ ), etc. Here and throughout, the set $\mathbb{R}^{n}$ denotes the $n$-dimensional real space with $\mathbb{S}^{n-1}$ denoting the set of unit vectors on $\mathbb{R}^{n}$.

\section{Metric constraint}

The fundamental idea of shape morphing is to write a heterogeneous pattern of director field on a thin sheet that when actuated creates a pattern of spontaneous stretch that is not compatible in the plane, thereby leading the sheet to a complex three-dimensional shape. This can be described using a metric constraint [1, 5, 22, that relates the three-dimensional deformation $\mathbf{y}$ of the midplane of a thin sheet $\omega \subset \mathbb{R}^{2}$ to the induced metric due to the actuation (described by a parameter $r \equiv r(\Delta T)>0)$ and a director pattern $\mathbf{n}_{0}: \omega \rightarrow \mathbb{S}^{2}$ :

$$
(\tilde{\nabla} \mathbf{y})^{T} \tilde{\nabla} \mathbf{y}=r^{-1 / 3}\left(\mathbf{I}_{2 \times 2}+(r-1) \tilde{\mathbf{n}}_{0} \otimes \tilde{\mathbf{n}}_{0}\right)=: \tilde{\ell}_{\mathbf{n}_{0}}
$$




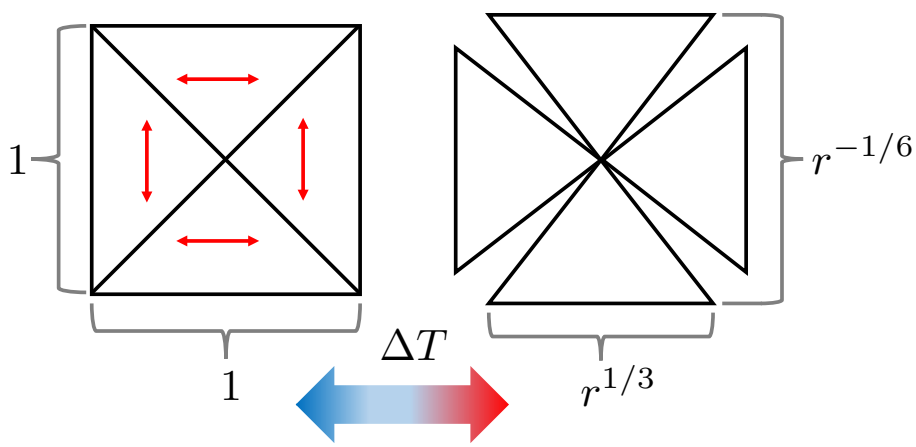

(a)
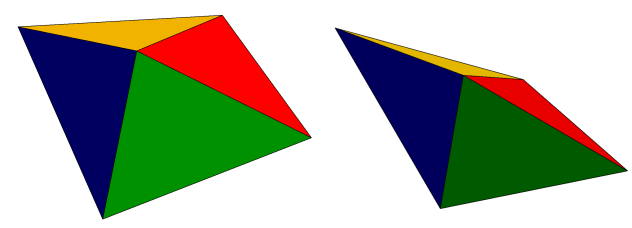

(c)

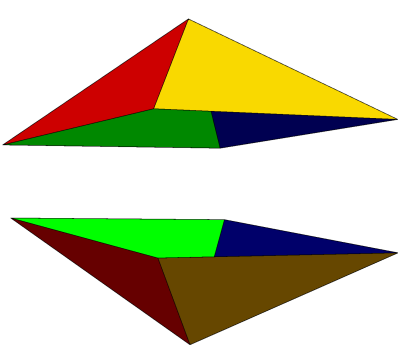

(b)

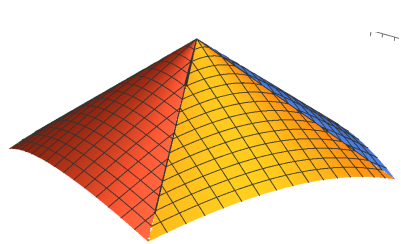

(d)

Figure 3: A simple example of a nonisometric origami. (a) The shape change induced by actuation of this patterned design cannot be accommodated by a planar deformation of the sheet. Alternatively, the sheet can form a pyramid to realize this shape change (metric). However, there are many degeneracies: (b) the pyramid can actuation up or down; (c) the folding angles need not be equal; (d) each triangle can curve so that the actuation is conical.

where $\tilde{\nabla}$ is the in-plane gradient and $\tilde{\mathbf{n}}_{0}:=\left(\mathbf{n}_{0} \cdot \mathbf{e}_{1}, \mathbf{n}_{0} \cdot \mathbf{e}_{2}\right) \in \mathbb{R}^{2}$ is the projection of the the director on to the plane. One can justify this rigorously [23] on the basis of energy minimization of the well-established free energy of nematic elastomers [2, 31]. Much of the work has focused on identifying specific patterns $\mathbf{n}_{0}$ that are consistent with interesting deformations (shapes) $\mathbf{y}$.

There are two broadly open issues. The first is degeneracy: there often are many deformations that satisfy the metric constraint (1) for a given director pattern. Indeed note that the composition of $\mathbf{y}$ with any isometric map $\mathbf{z}: \mathbf{y}(\omega) \rightarrow \mathbb{R}^{3}$ also satisfies (1). The second is the inverse problem: while interesting examples have been identified, a broad strategy to design complex shapes is yet to emerge. In this paper we propose a strategy that involves nonisometric origami where both the director and the deformation gradient are piecewise uniform to address both these issues.

\section{Nonisometric origami}

We introduce nonisometric origami through a simple example where a flat sheet is actuated into a pyramid. Consider a unit square sheet made of equal triangles of base 1 and height $1 / 2$ as depicted Figure 3(a). In each triangle the director is programmed in the plane and is parallel to the base of the triangle. Thus upon heating, each triangle desires a spontaneous contraction $r^{1 / 3}$ along its base and an expansion $r^{-1 / 6}$ along its height, consistent with (1). Notice, though, that it is not possible to satisfy this shape changing distortion in the plane without breaking up sheet along one of the interfaces distinguishing the four triangles. Alternatively, notice that by rotating each of these triangles out-of-plane, we can form a pyramid to accommodate the heterogeneous shape changing distortion while keeping the interfaces intact. That is, through the pyramid actuation, we obtain a deformation which satisfies the induced metric in (1). Thus, we realize a three-dimensional faceted 
shape by patterning the directors to be piecewise constant in polygonal regions. This is an example of nonisometric origami.

However, as we highlight with Figure $3(\mathrm{~b}-\mathrm{d})$, there is degeneracy in the shapes that can accommodate this induced metric: The pyramid can go up or down. Further, the folding angles do not all have to be equal, as the symmetric pyramid can be deformed without stretching the triangles into a range of pyramidal shapes. Finally, the faces do not have to be flat; instead each triangle can be deformed without stretch into a conical strip.

\section{Strategy to bias the actuation}

We seek a strategy to select amongst the various degenerate shapes, and the basic idea is relatively simple. These shapes all involve the same stretch, but differ by the curvature 1 Therefore, we can select a shape by a preferred curvature at each point. We do this by using a twisted nematic configuration. These profiles introduce a preferred spontaneous curvature upon actuation, but this requires careful study. Indeed, if the twist profile is not properly designed, then actuation can lead to anti-clastic bending (i.e., negative Gauss curvature) and, as a consequence, undesirable stretch.

Returning to our example of the pyramid, we introduce a twist nematic director profile in narrow regions surrounding the interfaces as depicted in Figure 4(a). To study one such interface, we fix a local right-hand frame $\left\{\mathbf{e}_{1}, \mathbf{e}_{2}, \mathbf{e}_{3}\right\} \in \mathbb{S}^{2}$ with $\mathbf{e}_{1}$ tangent to the interface and $\mathbf{e}_{3}$ out-of-plane, and we denote the three dimensional wedge domain as $\Omega_{h}:=\omega_{\text {wedge }} \times(-h / 2, h / 2)$ where $\omega_{\text {wedge }} \subset \mathbb{R}^{2}$ is as depicted and $h$ the thickness of the sheet. On $\Omega_{h}$, we set the director to vary through the thickness with an average orientation at a 45 degree angle from this interface, as depicted:

$$
\mathbf{n}_{0}^{h}(\mathbf{x})=\cos \left(\frac{\tau x_{3}}{h}\right)\left\langle\mathbf{n}_{0}\right\rangle\left(x_{2}\right)+\sin \left(\frac{\tau x_{3}}{h}\right)\left\langle\mathbf{n}_{0}\right\rangle^{\perp}\left(x_{2}\right),
$$

where $\left\langle\mathbf{n}_{0}\right\rangle\left(x_{2}\right):=(1 / \sqrt{2})\left(\mathbf{e}_{1}-\operatorname{sgn}\left(x_{2}\right) \mathbf{e}_{2}\right),\left\langle\mathbf{n}_{0}\right\rangle^{\perp}\left(x_{2}\right):=\operatorname{sgn}\left(x_{2}\right)\left(\mathbf{e}_{3} \times\left\langle\mathbf{n}_{0}\right\rangle\left(x_{2}\right)\right)$ and sgn: $\mathbb{R} \rightarrow$ $\{-1,1\}$ denotes the sign function with $\operatorname{sgn}(0)=1$. Notice that $\mathbf{n}_{0}^{h}(\tilde{\mathbf{x}}, h / 2)=\mathbf{n}_{0}^{t}\left(x_{2}\right)$ at the top of the sheet and $\mathbf{n}_{0}^{h}(\tilde{\mathbf{x}},-h / 2)=\mathbf{n}_{0}^{b}\left(x_{2}\right)$ at the bottom of the sheet. This director profile leads to a spontaneous stretch described by the square-root of the fully three-dimensional metric

$$
\begin{aligned}
\boldsymbol{\ell}_{\mathbf{n}_{0}^{h}}^{1 / 2}(\mathbf{x}) & :=r^{-1 / 6}\left(\mathbf{I}_{3 \times 3}+\left(r^{1 / 2}-1\right) \mathbf{n}_{0}^{h}(\mathbf{x}) \otimes \mathbf{n}_{0}^{h}(\mathbf{x})\right) \\
& =r^{-1 / 6}\left(\mathbf{I}_{3 \times 3}-\epsilon\left(\mathbf{A}_{S}\left(x_{2}\right)+\left(\tau x_{3} / h\right) \mathbf{A}_{B}+O\left(\tau^{2}\right)\right)\right),
\end{aligned}
$$

where the latter equality is obtained by a Taylor expansion in $\tau$, and

$$
\mathbf{A}_{S}\left(x_{2}\right)=\left\langle\mathbf{n}_{0}\right\rangle\left(x_{2}\right) \otimes\left\langle\mathbf{n}_{0}\right\rangle\left(x_{2}\right), \quad \mathbf{A}_{B}=\mathbf{e}_{1} \otimes \mathbf{e}_{1}-\mathbf{e}_{2} \otimes \mathbf{e}_{2}
$$

with $\epsilon=1-r^{1 / 2}$. Physically, $\mathbf{A}_{S}\left(x_{2}\right)$ describes the spontaneous in-plane stretch and $\mathbf{A}_{B}$ describes the spontaneous curvature. Notice that the latter term corresponds to principal curvatures of opposite signs, and this can lead to anti-clastic bending in the wedge (i.e., negative Gauss-curvature). Additionally, due to Gauss' Theorema Egregium, it is not possible for the wedge to have both uniform stretch and uniform negative Gauss-curvature. Thus, this twist nematic pattern will be internally stressed, and further analysis is required to understand the nature of equilibrium. The basic idea in what follows is that the anti-clastic bending can be suppressed completely in favor of singly-bent ridges if the wedge is sufficiently wide.

We first observe that the $r^{-1 / 6}$ in front for the preferred local stretch (3) can be dropped as this describes a homogeneous shape-change under actuation that does not influence the competition

\footnotetext{
${ }^{1}$ One should think of the folding angle in idealized origami actuation as a form of curvature.
} 


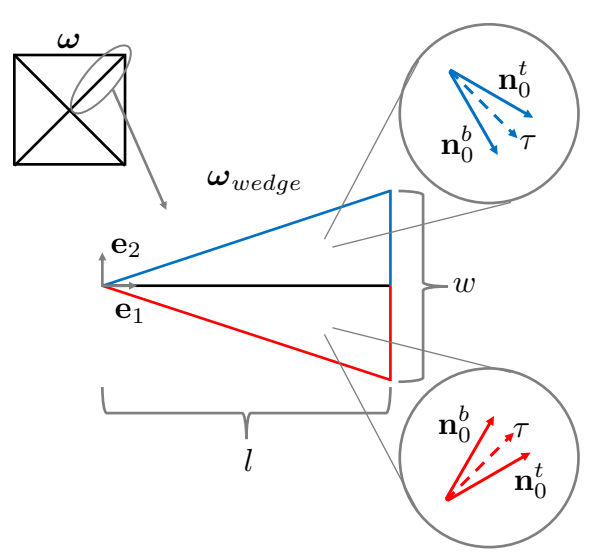

(a)

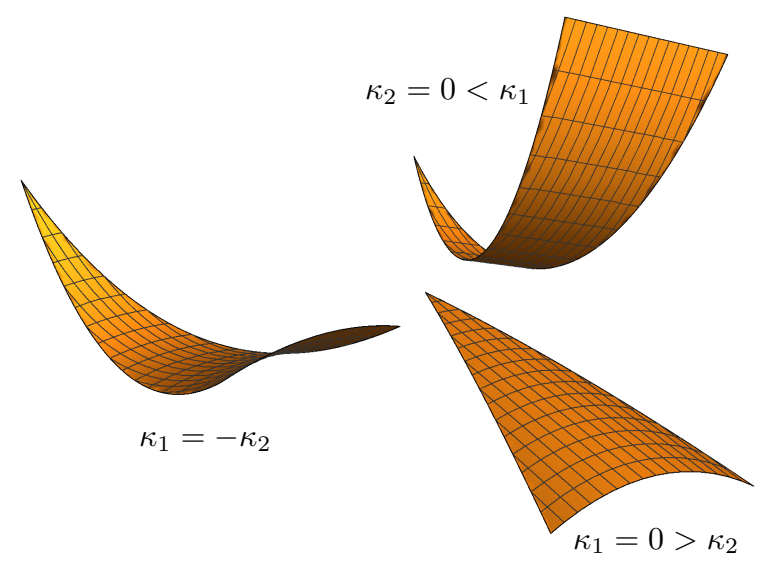

(b)

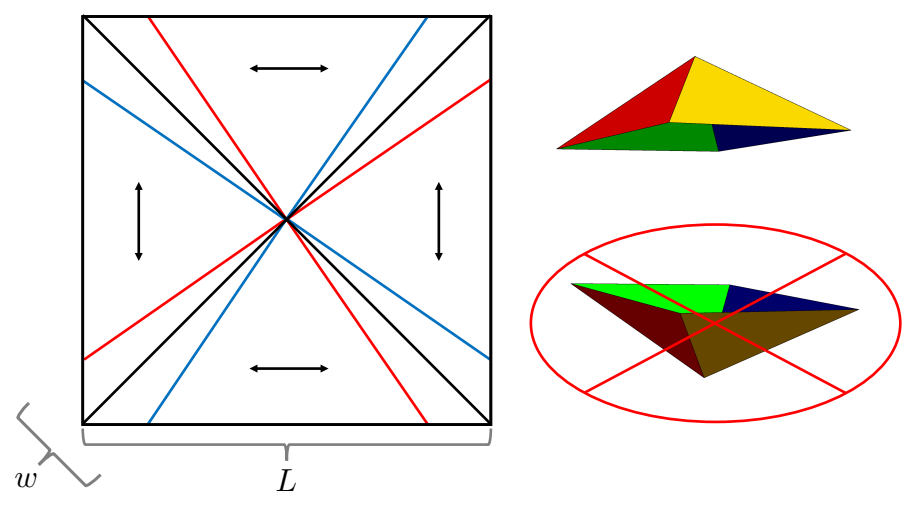

(c)

Figure 4: (a) The design for a twist nematic wedge at the interfaces of the nonisometric origami pattern. $\mathbf{n}_{0}^{t}$ denotes the director design at the top face of the wedge, and $\mathbf{n}_{0}^{b}$ the design at the bottom. (b) The twist profile in (a), when heated, can induce negative Gauss curvature $\left(\kappa_{1}=-\kappa_{2}>0\right)$, positive curvature along the interface $\left(\kappa_{1}>0=\kappa_{2}\right)$, or negative curvature transverse to the interface $\left(\kappa_{2}<0=\kappa_{1}\right)$. This depends on the geometry $(\tau, w, l)$ depicted as well as the thickness $h$ and the actuation parameter $\epsilon=1-r^{1 / 2} \in(0,1)$. (c) If designed carefully, then wedges, integrated into the nonisometric origami design, can bias the actuation.

between stretching and bending. We then start from the total energy (according to the wellestablished free energy of nematic elastomers [2, 31]), and study the asymptotics of this energy in thickness under the assumption that we have moderate stretch or $\epsilon \sim h^{2}$ (similar to [13]). We obtain a von Kármán theory described by the energy

$$
\begin{aligned}
& \mathcal{E}_{v K}(\tilde{\mathbf{u}}, v)= \\
& \quad \int_{0}^{l} \int_{-\frac{w x_{1}}{2 l}}^{\frac{w x_{1}}{2 l}}\left(Q_{2}\left(\operatorname{sym} \tilde{\nabla} \tilde{\mathbf{u}}+\frac{1}{2} \tilde{\nabla} v \otimes \tilde{\nabla} v+\epsilon \tilde{\mathbf{A}}_{S}\left(x_{2}\right)\right)+\frac{h^{2}}{12} Q_{2}\left(\tilde{\nabla} \tilde{\nabla} v-\frac{\epsilon \tau}{h} \tilde{\mathbf{A}}_{B}\right)\right) d x_{1} d x_{2},
\end{aligned}
$$

where $(\tilde{\mathbf{u}}, v): \omega_{\text {wedge }} \rightarrow \mathbb{R}^{2} \times \mathbb{R}$ are the in-plane and out-of-plane displacements of the wedge, respectively, $Q_{2}(\tilde{\mathbf{F}})=\mu\left(|\tilde{\mathbf{F}}|^{2}+(\operatorname{Tr} \tilde{\mathbf{F}})^{2}\right)$ with $\mu>0$ the shear modulus of the elastomer, and 
$\tilde{\mathbf{A}}_{S}\left(x_{2}\right)$ and $\tilde{\mathbf{A}}_{B}$ are the $2 \times 2$ principal minors of the tensors in (4).

To minimize this energy, we set $\tilde{\mathbf{u}}(\tilde{\mathbf{x}})=\tilde{\mathbf{u}}^{*}(\tilde{\mathbf{x}})+\epsilon\left(\tilde{\mathbf{x}}+\left|x_{2}\right| \tilde{\mathbf{e}}_{1}\right)$. Notice that the latter term corresponds jump in the strain at the interface and satisfies the (linearized) metric constraint $\operatorname{sym} \tilde{\nabla}\left(\epsilon\left(\tilde{\mathbf{x}}+\left|x_{2}\right| \tilde{\mathbf{e}}_{1}\right)\right)=-\epsilon \tilde{\mathbf{A}}_{S}\left(x_{2}\right)$. We then minimize the energy amongst the class of smooth polynomial displacement: 2

$$
v(\tilde{\mathbf{x}})=\frac{\epsilon \tau}{h}\left(\kappa_{1} x_{1}^{2}+\kappa_{2} x_{2}^{2}\right), \quad \tilde{\mathbf{u}}^{*}(\tilde{\mathbf{x}})=\left(\begin{array}{c}
b_{1} x_{1}^{3}+b_{2} x_{1} x_{2}^{2}+b_{3} x_{1} \\
c_{1} x_{2}^{3}+c_{2} x_{2} x_{1}^{2}+c_{3} x_{2}
\end{array}\right) .
$$

Minimizing out the coefficients associated with the in-plane displacements, we find that

$$
\min _{\left(b_{i}, c_{i}\right)} \mathcal{E}_{v K}(\tilde{\mathbf{u}}, v)=\frac{\mu}{12} l w \epsilon^{2} \theta^{2}\left(q\left(\kappa_{1}, \kappa_{2}\right)+g_{f} \kappa_{1}^{2} \kappa_{2}^{2}\right)
$$

where $q\left(\kappa_{1}, \kappa_{2}\right)=1+2 \kappa_{2}\left(\kappa_{2}+1\right)+2\left(\kappa_{1}+\kappa_{2}\right)^{2}+2 \kappa_{1}\left(\kappa_{1}-1\right)$ and

$$
g_{f}=\left(\frac{l^{4}}{l^{4}+(2 / 15) l^{2} w^{2}+(7 / 240) w^{4}}\right)\left(\frac{1}{15} \frac{\epsilon^{2} \tau^{2}}{(h / w)^{4}}\right) .
$$

Since $q\left(\kappa_{1}, \kappa_{2}\right)$ is minimized at $\kappa_{1}=1 / 2=-\kappa_{2}$, the first term in (7) prefers the deformation of the wedge to be anti-clastic bending. In contrast, the second term in this energy prefers that one of the curvatures (either $\kappa_{1}$ or $\kappa_{2}$ ) is zero. The balance is determined by the factor $g_{f}$ which embeds all the design variables $(\epsilon, \tau, h, w, l)$ into a single parameter. Thus, this factor $g_{f}$ provides a means of rationally designing the interfaces of the origami to achieve bias in their bending.

Specifically, if $g_{f}<384$, then the unique equilibrium is anti-clastic bending $\kappa_{1}\left(g_{f}\right)=-\kappa_{2}\left(g_{f}\right) \in$ $(1 / 8,1 / 2]$. However, if $g_{f}>384$, two equivalent lower energy states emerge: $0<-\kappa_{2}\left(g_{f}\right)<1 / 8<$ $\kappa_{1}\left(g_{f}\right)<1 / 4$ or $0<\kappa_{1}\left(g_{f}\right)<1 / 8<-\kappa_{2}\left(g_{f}\right)<1 / 4$. Further, for $g_{f} \gg 384,-\kappa_{2}\left(g_{f}\right) \approx 0$ and $\kappa_{1}\left(g_{f}\right) \approx 1 / 4$ (or the opposite happens). Thus, if $g_{f}$ is designed to be large enough, then negative Gauss curvature is suppressed, and the wedge prefers being singly bent.

\section{Pyramid by design}

We now apply this idea to the pyramid with a design shown schematically in Figure 4(c). We assume that the area of the wedge is small compared to the overall area of the pyramid. If $g_{f} \gg 384$, the wedges in isolation favor being singly bent with either $\kappa_{2} \approx 0<\kappa_{1}$ or $\kappa_{1} \approx 0>\kappa_{2}$ as depicted in 4 (b). Notice that the latter of these two configurations forms natural ridge consistent with the actuation of pyramid along an interface. In contrast, the former imparts curvature along the interface and this is not consistent with the overall metric constraint. Therefore, if $g_{f} \gg 384$, the latter configuration $\left(\kappa_{1} \approx 0>\kappa_{2}\right)$ is preferred when these integrated into the nonisometric origami design. This breaks the up-down symmetry. Further, the ridges prefer curvature while the faces prefer to be flat. Therefore, properly designed, we expect a symmetric pyramid with flat faces.

We examine this experimentally in Figure 5. We consider square sheet of length $L=2 \mathrm{~cm}$ and thickness $h=30 \mu \mathrm{m}$. We consider a nematic elastomer with the anisotropy parameter $\epsilon=1-r^{-1 / 2}$ in the range of $[.2, .4]$, depending on the temperature of the hotplate and the cross-linking density of the sample. Thus, we design the bias so that $\tau=\pi / 12$ and width of the wedge is $w \approx 4 \mathrm{~mm}$. Note that $l \gg w$, and so for these parameters, $g_{f} \approx 50000 \gg 3843^{3}$ The measured shape on heating the sheet is shown. It actuates up, the faces are flat and the slope is as expected.

\footnotetext{
${ }^{2}$ The terms absent to leading order in this expansion vanish identically when accounted for in the energy minimization.

${ }^{3}$ We have taken $\epsilon=0.2$ in the calculation of $g_{f}$ to be conservative.
} 


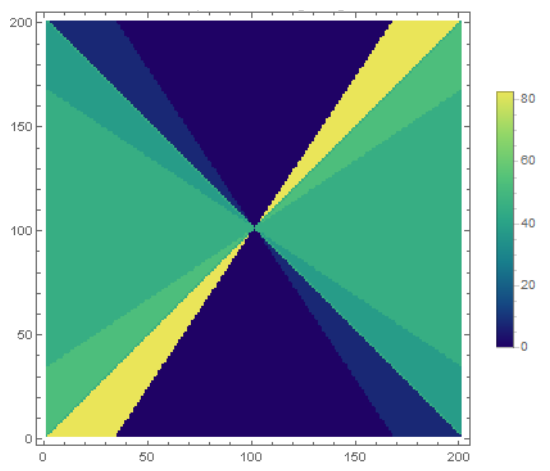

(a)

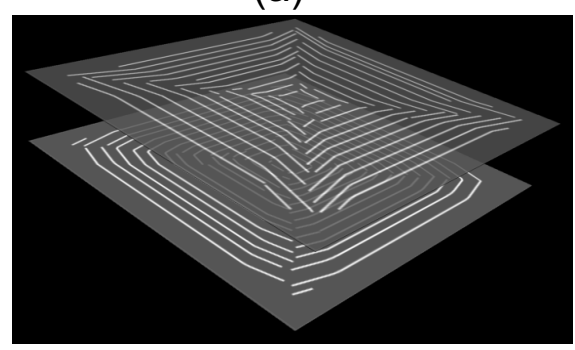

(b)

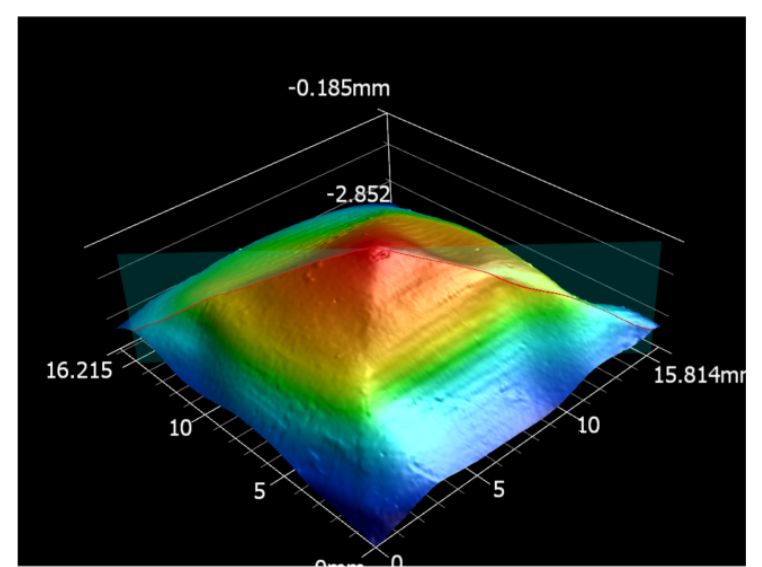

(c)

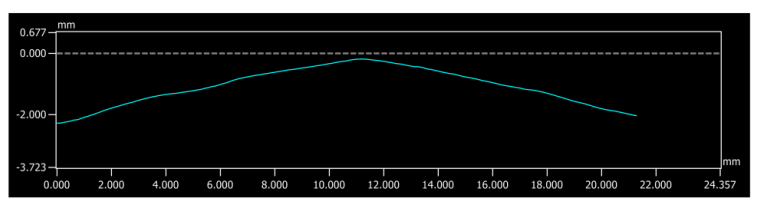

(d)

Figure 5: Experiment to realize a pyramid by design: (a) The stage angle for photo-alignment at the bottom surface. (b) Contour lines tangent to the director field for this design. (c) The actuated shape (the colors indicate elevation). (d) The pyramidal deformation for the cross sectional slice shown in (c).

\section{Nonisometric origami as building blocks}

We now show how to link simple nonisometric origami patterns together as building blocks to generate complex shape. For the the efficacy of this design strategy, our ability to bias the simple building blocks to achieve a unique actuation proves crucial. We explain this by comparing experiments of designs composed of both biased and unbiased nonisometric origami building blocks.

We first consider the simplest possible design composed of building blocks of unbiased nonisometric origami. This is given in Figure 2. The design is that of two pyramid patterns linked across a region where the director is identical for each pattern. This yields a compatible design (i.e., a design which admits deformations satisfying the metric constraint (1)) because the desired spontaneous shape change across this linkage is identical.

In particular, the sample in 2(a) is patterned uniformly in the region where the building blocks are linked 4 Thus importantly, we do not expect the sample, upon actuation, to deform as if there is a sharp interface in this region (such an interface would result in bending stresses that are completely unnecessary). Rather, we expect the deformation to be uniform here, and consequently, we expect the total deformation to be a complex three dimensional shape which accommodates this region of uniform deformation. Simply put, we expect that the pyramids do not deform in isolation but rather as an integrated structure. These expectations are exactly realized in the experiments shown in Figure 2(b).

We describe this in more detail: The initially flat sample shown in 2(a) is placed on top of a hot plate $T \sim 150 C$, and it actuates into one of the two configurations shown in 2(b). It appears

\footnotetext{
${ }^{4}$ the grey dashed line in the figure is simply delineates the building blocks.
} 
(a)

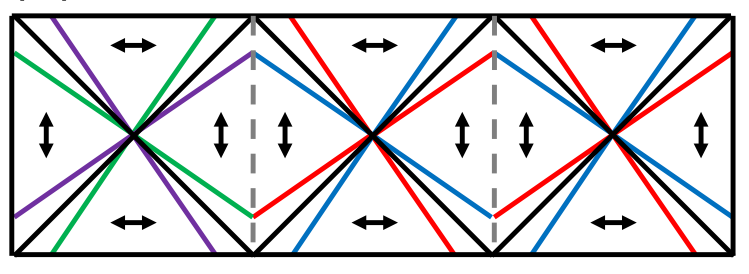

(b)

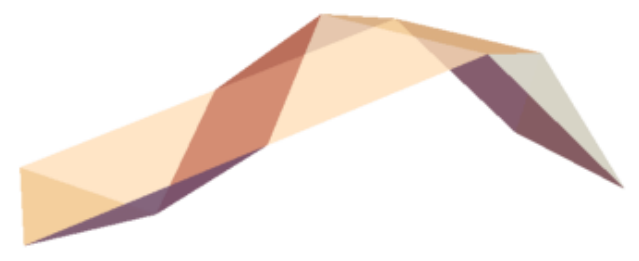

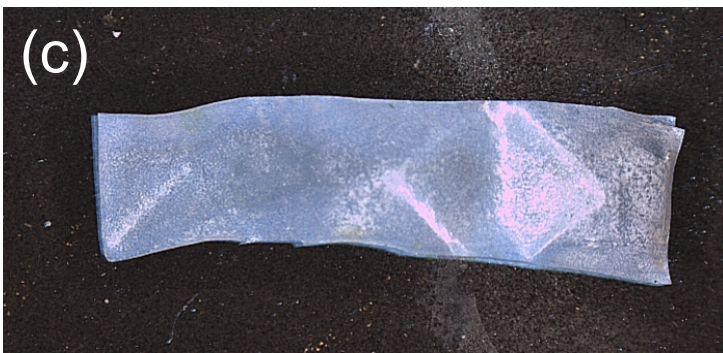

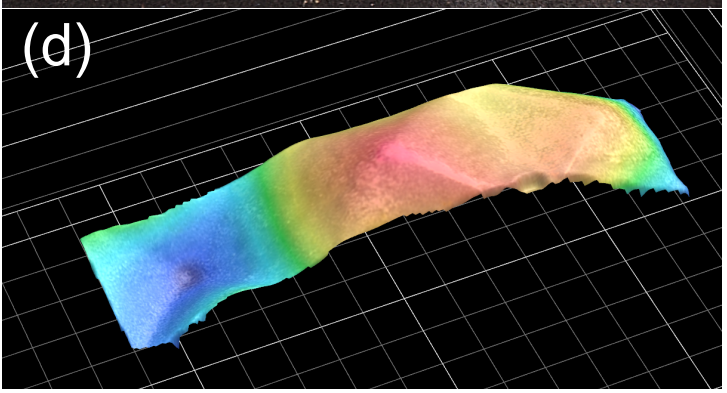

Figure 6: Programmable actuation bias is demonstrated in a set of three joined pyramidal junctions. (a) A schematic of the design. For the center and right wedges, the twist is as in Figure 4(a). For the left, the top and bottom director prescription is reversed (this is emphasized with the change in color). (b) Intended shape, in which one pyramid actuates down and the remaining two actuate up. The sheet is taken to rest on a flat surface. (c) Top view of fabricated monolithic sample, patterned with three of the biased pyramidal building blocks from Figure 5. Each building block is $5 \mathrm{~mm} \times 5 \mathrm{~mm}$. (d) Measured shape of sample upon heating shows that each pyramid actuates in its programmed direction. False color represents heights, with a span of $3 \mathrm{~mm}$.

to be bi-stable under the actuation as both configurations are observed in the single sample shown, with little discernible preference for one or the other. Specifically, the choice of actuation seems governed only by how the sample is manipulated when placed on the hot plate. Further, once all external manipulation (from the experimenter) ceases, the actuated configuration is robust in the sense that it maintains its three dimensional shape on the heated hot-plate.

In comparing this result with the theory, recall again that the building blocks in Figure 3(a) can actuate into a pyramid going up or down (i.e., 3(b)) under the metric constraint since we have not used twisted interfaces. For the patterned sheet in 2 (a), if both of the building blocks attempt to realize the symmetric pyramid actuation $5^{5}$, then there are exactly two non-trivial solutions that avoid bending the interface in which the building blocks are merged (i.e., the gray-dashed line in 2(a)): One occurs if both pyramids actuate up (or down), in which case the pyramids must rotate over to keep the deformation of the initially square region uniform as in the top of 2 (c). The other configuration occurs if one pyramid actuates up and the other down as in the bottom of 2(c). Both configurations are completely equivalent in this theoretical framework. They are also exactly the bi-stable configurations observed in the experiments $2(\mathrm{~b})$.

This success notwithstanding, one can never hope to achieve a specific desired shape through patterns of unbiased building blocks. Indeed, the up-down symmetry of the building blocks enables

\footnotetext{
${ }^{5}$ One can argue against the other shapes in 3(c) and (d). Indeed, non-symmetric pyramids as in (c) likely have higher bending energy then their symmetric counterpart. This is certainly the case if bending energy can be captured by a strictly convex and increasing function of the folding angle magnitudes. In addition, conical shapes as in (d) cannot be linked together compatibly.
} 


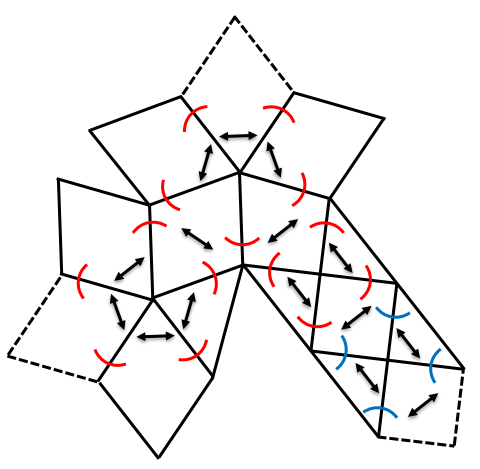

(a)

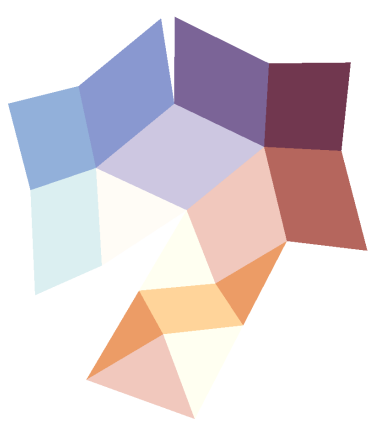

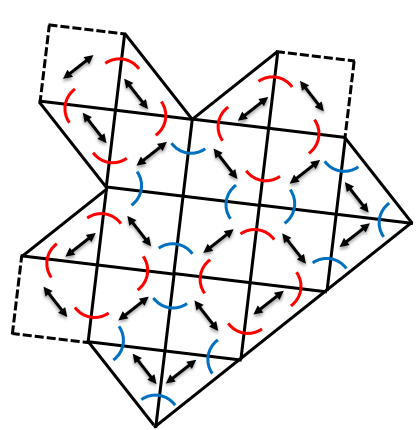

(b)

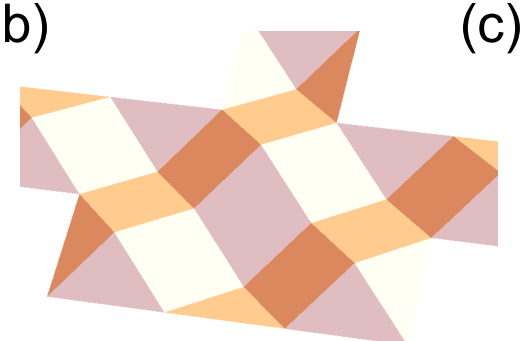

(c)
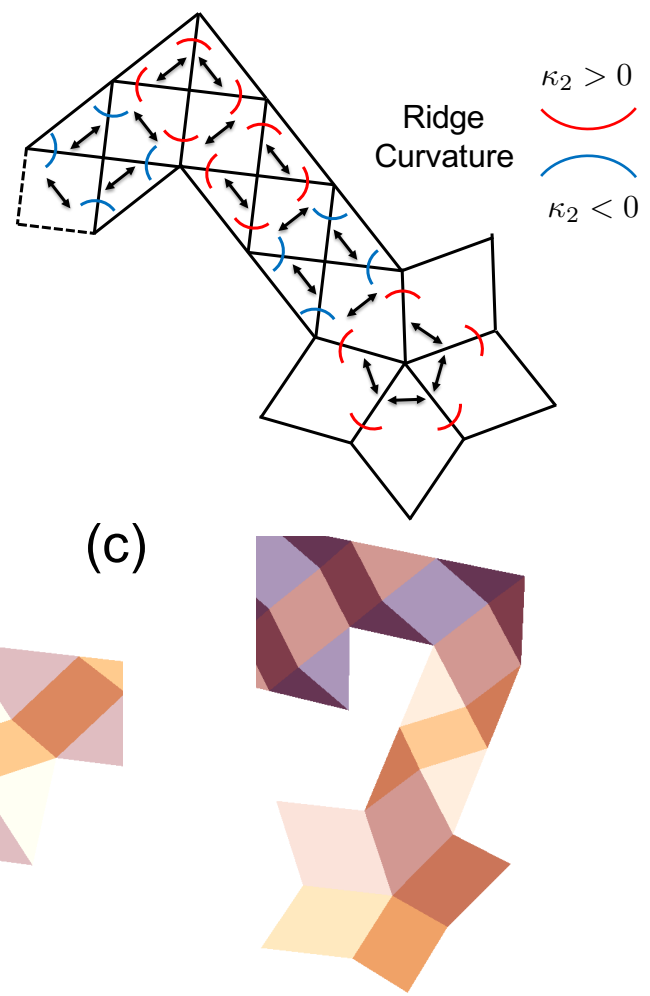

Figure 7: Designing a humanoid soft robot by linking symmetric four-faced and five-faced building blocks. The figure described the design and actuation of (a) a portion of the head and neck region, (b) the body region, and (c) one of the arms. The colored arcs represent the designed curvature along the ridges of the origami; blue for a twist nematic wedge design to induce negative curvature transverse to the ridge, and red for a wedge design to induce positive curvature.

a multi-stable landscape of possible actuated configurations, and many of these will not be of any interest to the designer. However, biased building blocks offer a route to systematically achieve a specified shape.

To highlight this, we now modify the design in 2(a) by composing together three simple but biased pyramid building blocks as depicted in Figure 6(a). For each building block, we prescribe twist nematic regions along the interfaces. These are designed to break the up-down symmetry of the pyramid actuation, while ensuring that unwanted anti-clastic bending is suppressed. Specifically, the center and right building block are designed to actuate up into a pyramid, while the left is designed to actuate down. For the latter, we simply flip the top and bottom prescription of the director to achieve the opposite bias. This design, in theory, results in a unique energetically favorable actuation described by 6(b) (this is simply a combination of the two actuations in 2(c)). The corresponding experiment in 6(c-d) is in excellent agreement: The pyramids are biased, and the entire structure rotates over during the actuation to avoid bending the faces of uniform director. In doing all this, the patterned sheet achieves the predicted origami actuation.

\section{Designing complex shapes}

We have demonstrated experimentally three capabilities in actuating patterned nematic elastomer sheets: 1) Predictable three dimensional origami shape can be realized through the design of non- 
isometric origami building blocks. 2) These building blocks, when linked together, can be used to produce complex multi-stable, yet predictable, actuations. 3) The inherent degeneracies of these origami patterns can be suppressed, and a unique actuation can be achieved by designing bias at the ridges of the origami.

This functionality enables a design landscape with striking possibilities. We showcase this through a pattern to achieve, upon heating, the "humanoid" soft robot shown in Figure 1. The design is composed of symmetric four and five-faced building blocks. Each of the 27 total building blocks is independently compatible, and these are linked across regions in which the director profile is uniform. For example, in the head and neck region depicted in Figure 7(a), we have two such linkages - one where a five-faced building blocks merges with a four-faced building block to form part of the neck and another where two five-faced building blocks merge to build a portion of the head. To ensure that the design actuates the desired shape, we have designed a bias at each of the interfaces. The blue arcs describe an appropriately designed wedge whose twist profile is prescribed at the top and bottom of the wedge as depicted in Figure 4(a). This leads to symmetric pyramids which actuates up just as in 4(c). In contrast, the red arcs describe a wedge design 4(a) in which the top and bottom directors of the twist profile are reversed. This leads to symmetric pyramids which actuate down. By controlling the shape and direction of actuation for these simple nonisometric origami building blocks and linking these together compatibly, we are able to systematically build a design to achieve the desired humanoid shape.

\section{Materials and methods}

Patterned LCE films are fabricated following procedures described in [12, 27]. In short, glass slides are spin-coated with a commercial photoalignment material (PAAD-22, BEAM Co). Photoalignment is performed by sequential exposures to a focused $445 \mathrm{~nm}$ laser spot. By adjusting the laser's linear polarization at each successive exposure location, we build up the desired complex alignment pattern. This pattern is then permanently fixed by spin-coating with a thin layer of mesogenic monomer (RM257, Synthon, 3 wt solution in dichloromethane) and flood-curing. Finally, two slides are glued together to form an alignment cell, using glass beads to ensure a 50 micron cell spacing.

A previously reported one-pot LCE formulation [28] is then capillary filled into the alignment cell in the isotropic phase. Upon cooling to the nematic phase, it is flood-cured to form a freestanding monolithic elastomer film. Heat-stimulated shape change is quantitatively measured using a structured-illumination optical 3D scanner with micron-scale height resolution (Keyence VR-3200).

\section{References}

[1] H. Aharoni, E. Sharon, and R. Kupferman. Geometry of thin nematic elastomer sheets. Physical review letters, 113(25):257801, 2014.

[2] P. Bladon, E. Terentjev, and M. Warner. Transitions and instabilities in liquid crystal elastomers. Physical Review E, 47(6):R3838, 1993.

[3] L. T. de Haan, C. Sánchez-Somolinos, C. M. Bastiaansen, A. P. Schenning, and D. J. Broer. Engineering of complex order and the macroscopic deformation of liquid crystal polymer networks. Angewandte Chemie International Edition, 51(50):12469-12472, 2012.

[4] E. Efrati, E. Sharon, and R. Kupferman. Elastic theory of unconstrained non-euclidean plates. Journal of the Mechanics and Physics of Solids, 57(4):762-775, 2009. 
[5] E. Efrati, E. Sharon, and R. Kupferman. The metric description of elasticity in residually stressed soft materials. Soft Matter, 9(34):8187-8197, 2013.

[6] S. Felton, M. Tolley, E. Demaine, D. Rus, and R. Wood. A method for building self-folding machines. Science, 345(6197):644-646, 2014.

[7] K. Fuchi, T. H. Ware, P. R. Buskohl, G. W. Reich, R. A. Vaia, T. J. White, and J. J. Joo. Topology optimization for the design of folding liquid crystal elastomer actuators. Soft matter, 11(37):7288-7295, 2015.

[8] V. Gimenez-Pinto, F. Ye, B. Mbanga, J. V. Selinger, and R. L. Selinger. Modeling out-of-plane actuation in thin-film nematic polymer networks: From chiral ribbons to auto-origami boxes via twist and topology. Scientific Reports, 7, 2017.

[9] E. Hawkes, B. An, N. M. Benbernou, H. Tanaka, S. Kim, E. Demaine, D. Rus, and R. J. Wood. Programmable matter by folding. Proceedings of the National Academy of Sciences, 107(28):12441-12445, 2010.

[10] D.-H. Kim and J. A. Rogers. Stretchable electronics: materials strategies and devices. Advanced Materials, 20(24):4887-4892, 2008.

[11] Y. Klein, E. Efrati, and E. Sharon. Shaping of elastic sheets by prescription of non-euclidean metrics. Science, 315(5815):1116-1120, 2007.

[12] B. A. Kowalski, V. P. Tondiglia, T. Guin, and T. J. White. Voxel resolution in the directed self-assembly of liquid crystal polymer networks and elastomers. Soft Matter, 2017.

[13] M. Lewicka, L. Mahadevan, and M. R. Pakzad. The föppl-von kármán equations for plates with incompatible strains. In Proceedings of the Royal Society of London A: Mathematical, Physical and Engineering Sciences, volume 467, pages 402-426. The Royal Society, 2011.

[14] C. Majidi. Soft robotics: a perspective - current trends and prospects for the future. Soft Robotics, 1(1):5-11, 2014.

[15] C. Modes, K. Bhattacharya, and M. Warner. Gaussian curvature from flat elastica sheets. In Proceedings of the Royal Society of London A: Mathematical, Physical and Engineering Sciences, volume 467, pages 1121-1140. The Royal Society, 2011.

[16] C. Modes, M. Warner, C. Sánchez-Somolinos, L. De Haan, and D. Broer. Mechanical frustration and spontaneous polygonal folding in active nematic sheets. Physical Review E, 86(6):060701, 2012 .

[17] C. D. Modes, K. Bhattacharya, and M. Warner. Disclination-mediated thermo-optical response in nematic glass sheets. Physical Review E, 81(6):060701, 2010.

[18] C. D. Modes and M. Warner. Blueprinting nematic glass: Systematically constructing and combining active points of curvature for emergent morphology. Physical Review E, 84(2):021711, 2011.

[19] G. N. Mol, K. D. Harris, C. W. Bastiaansen, and D. J. Broer. Thermo-mechanical responses of liquid-crystal networks with a splayed molecular organization. Advanced Functional Materials, 15(7):1155-1159, 2005. 
[20] C. Mostajeran. Curvature generation in nematic surfaces. Physical Review E, 91(6):062405, 2015.

[21] C. Mostajeran, M. Warner, T. H. Ware, and T. J. White. Encoding gaussian curvature in glassy and elastomeric liquid crystal solids. In Proc. R. Soc. A, volume 472, page 20160112. The Royal Society, 2016.

[22] P. Plucinsky, M. Lemm, and K. Bhattacharya. Programming complex shapes in thin nematic elastomer and glass sheets. Physical Review E, 94(1):010701, 2016.

[23] P. Plucinsky, M. Lemm, and K. Bhattacharya. Actuation of thin nematic elastomer sheets with controlled heterogeneity. Archive for Rational Mechanics and Analysis, 2017.

[24] J. Rogers, Y. Huang, O. G. Schmidt, and D. H. Gracias. Origami mems and nems. Mrs Bulletin, 41(2):123-129, 2016.

[25] Y. Sawa, F. Ye, K. Urayama, T. Takigawa, V. Gimenez-Pinto, R. L. Selinger, and J. V. Selinger. Shape selection of twist-nematic-elastomer ribbons. Proceedings of the National Academy of Sciences, 108(16):6364-6368, 2011.

[26] E. Sharon and E. Efrati. The mechanics of non-euclidean plates. Soft Matter, 6(22):5693-5704, 2010 .

[27] T. H. Ware, M. E. McConney, J. J. Wie, V. P. Tondiglia, and T. J. White. Voxelated liquid crystal elastomers. Science, 347(6225):982-984, 2015.

[28] T. H. Ware, Z. P. Perry, C. M. Middleton, S. T. Iacono, and T. J. White. Programmable liquid crystal elastomers prepared by thiol-ene photopolymerization. ACS Macro Letters, 4(9):942946, 2015.

[29] M. Warner, C. Modes, and D. Corbett. Curvature in nematic elastica responding to light and heat. In Proceedings of the Royal Society of London A: Mathematical, Physical and Engineering Sciences, volume 466, pages 2975-2989. The Royal Society, 2010.

[30] M. Warner, C. Modes, and D. Corbett. Suppression of curvature in nematic elastica. In Proceedings of the Royal Society of London A: Mathematical, Physical and Engineering Sciences, volume 466, pages 3561-3578. The Royal Society, 2010.

[31] M. Warner and E. M. Terentjev. Liquid crystal elastomers, volume 120. OUP Oxford, 2003.

[32] T. J. White and D. J. Broer. Programmable and adaptive mechanics with liquid crystal polymer networks and elastomers. Nature materials, 14(11):1087, 2015.

[33] S. Xu, Z. Yan, K.-I. Jang, W. Huang, H. Fu, J. Kim, Z. Wei, M. Flavin, J. McCracken, R. Wang, et al. Assembly of micro/nanomaterials into complex, three-dimensional architectures by compressive buckling. Science, 347(6218):154-159, 2015. 